OPEN ACCESS

Edited by:

Volker Scheer,

Fondation de la Science des Sports

Ultra, France

Reviewed by: José Ramón Alvero Cruz,

University of Málaga, Spain

Robinson Ramirez-Vélez,

Public University of Navarre, Spain

${ }^{*}$ Correspondence:

Shawnda A. Morrison shawnda.morrison@fsp.uni-lj.si orcid.org/0000-0003-3445-330X

Specialty section: This article was submitted to

Exercise Physiology,

a section of the journal

Frontiers in Physiology

Received: 21 December 2020 Accepted: 16 February 2021

Published: 08 March 2021

Citation:

Morrison SA, Sember $V$, Leskošek B, Kovač M, Jurak $G$ and

Starc G (2021) Assessment of Secular Trends and Health Risk in Pediatric Cardiorespiratory Fitness

From the Republic of Slovenia.

Front. Physiol. 12:644781. doi: 10.3389/fphys.2021.644781

\section{Assessment of Secular Trends and Health Risk in Pediatric Cardiorespiratory Fitness From the Republic of Slovenia}

\author{
Shawnda A. Morrison*, Vedrana Sember, Bojan Leskošek, Marjeta Kovač, Gregor Jurak \\ and Gregor Starc
}

Faculty of Sport, University of Ljubljana, Ljubljana, Slovenia

Objectives: Determine the temporal trends in cardiorespiratory fitness (CRF) and health risk of Slovenian schoolchildren across a 20 -year span, assessed via $20-\mathrm{m}$ shuttle run (20mSRT), including defining centile ranges and possible health risk(s) for each generation.

Methods: Nationally representative data from 9,426 healthy schoolchildren (6-14 years old) were used to determine changes in CRF across three generations, in 1993 $(n=3,174), 2003(n=3,457)$ and $2013(n=2,795)$ from a multistage, stratified, decennial study.

Results: 20mSRT performance declined $\sim 2.8 \%$ from 1993 to 2003, independent of age or sex of the child. This trend was reversed in 2013 , increasing by $\sim 8.2 \%$ across all age groups, for both girls and boys, for a net increase of $5.4 \%$. The magnitude of improvement was similar for both sexes. Moreover, girls in the 2013 generation (for ages 10-13 year) completed more stages than their 2003 male counterparts. Across all generations, children achieved CRF values corresponding to low cardiovascular risk for future health outcomes. Centile values ranged from "low" to "very high" depending on age, sex, and generation of the sample.

Conclusion: Negative trends in CRF from Slovenian schoolchildren were reversed by 2013 , indicating that Slovenia should continue implementing progressive national physical fitness strategies introduced between sampling periods (i.e., 2003-2013). Additionally, due to the universal nature of Slovenian schoolchildren achieving "healthy cut-off values" for 20mSRT (generation-inclusive), it is suggested that more specific cut-off criteria are developed, especially for younger children, and girls, so that future CRF results can be more accurately applied for both clinical and pedagogical users.

Keywords: health risk, generation, shuttle run, temporal trends, population health, youth 


\section{INTRODUCTION}

The 20-m shuttle run test (20mSRT), is an indirect measure of cardiorespiratory fitness (CRF) and one of the most widely used field measures of CRF, especially amongst children and youth (Lang et al., 2018b). Briefly, the 20mSRT involves continuous running back and forth between lines $20-\mathrm{m}$ apart, in time to audio signals. It consists of multiple stages which last $\sim 1 \mathrm{~min}$ and each stage comprises of a given number of laps. With every stage progression, the required running speed increases until volitional fatigue or the participant no longer completes the distance in-line with the audio signal on two consecutive occasions. There are multiple variations on the original Léger protocol (Léger et al., 1984, 1988), although the most widely-reported one remains the original. Worldwide secular trends in 20mSRT performance, and issues surrounding protocol standardization, have been reviewed in detail elsewhere (Tomkinson et al., 2003, 2019b; Olds et al., 2006). More recent literature has emphasized the importance of what the $20 \mathrm{mSRT}$ test can, and cannot, measure effectively (reviewed in Tomkinson et al., 2019a). Indeed, at its core the 20mSRT is not a direct measure of peak oxygen consumption $\left(\mathrm{V} \mathrm{O}_{2 \text { peak }}\right)$ and as such, is not able to comment on the exact amount of oxygen a moving body can take in, transport and use. Some authors have criticized the test's ability to adequately estimate CRF, instead suggesting it better estimates body fatness (Armstrong and Welsman, 2019; Welsman and Armstrong, 2019), although this argument does not appear to consider that the 20mSRT can be an appropriate predictor of future health in its own right (García-Hermoso et al., 2020), and a suitable marker of current health status (Ramírez-Vélez et al., 2017; Lang et al., 2018a), independent of adiposity (Machado-Rodrigues et al., 2014). Thus, the 20mSRT remains a useful and widely accessible test for population health research, especially when it is conducted in a standardized way, and provided the resultant measurements are interpreted within the scope of the tool.

A systematic review and meta-analysis determined that children and youth who fall below certain CRF cut-off points are at increased risk of cardiovascular disease (Ruiz et al., 2016). The comprehensive study included 9,280 children from 14 countries, aged 8 to 19 year, with $49 \%$ girls, and report $\mathrm{VO}_{2 \text { peak }}$ cut-off point $\leq 35$ and $\leq 42 \mathrm{~mL} \cdot \mathrm{kg}^{-1} \cdot \mathrm{min}^{-1}$ for girls and boys, respectively, who are deemed to be at greater health risk (Ruiz et al., 2016). The authors determined a $20 \mathrm{mSRT}$ running speed of $9.0 \mathrm{~km} / \mathrm{h}$ as an optimal cut-off point for detecting abdominal obesity in both sexes, across all ages (based on the original Léger protocol). The global, age-standardized prevalence of child obesity continues to increase from 0.7 to $0.9 \%$ (mean range) in 1975 to $4.8-9.1 \%$ (mean range) in 2016 (NCD Risk Factor Collaboration [NCDRisC], 2017), although Slovenia has largely reversed this trend in both sexes from 2009 to 2020 (Sorić et al., 2020) (notably before the COVID-19 crisis). It remains whether positive trends would also be observed for objective measures of physical fitness, including cardiorespiratory health.

Thus, the purpose of this study was two-fold, (1) to identify any directional trends in CRF for Slovenian schoolchildren and (2) to determine how Slovenian schoolchildren compare to international health standards. Based on worldwide CRF data trends, it was hypothesized that CRF would decrease from 1993 to 2013 consistent across age, in both boys and girls.

\section{MATERIALS AND METHODS}

Data were collected within the ACDSi study, approved by the Slovenian National Medical Ethics Committee (ID: 138/05/13), following the Declaration of Helsinki. All data are anonymous.

\section{Patient and Public Involvement}

Written, informed consent was obtained from parents or legal guardians of all children before voluntary participation; children could withdraw from the study, in whole or in part, anytime they wished. Schools, parents and children are kept informed of study progress through various communication methods (e.g., web pages, regular presentations at schools, etc.) where they could provide feedback on their experiences.

\section{Study Population}

The ACDSi study investigates children's biological, psychological and social development, described in detail elsewhere (Jurak et al., 2013). It is a cross-sectional decennial study that includes 11 primary schools. The ACDSi has been repeated four times since 1970/71; first in 1983 (Šturm and Strel, 1984) and every decade thereafter [1993 (Strel et al., 1996), 2003 (Kovač et al., 2004) and 2013 (Jurak et al., 2013)]. A national, representative sample was selected using a multi-stage, stratified design. Ten research sites were selected according to four Slovenian settlement types (village, rural town, industrial town, and city) and regions. One primary school was selected from each project site, except in the capital region where an additional school was added in 1993, in order to meet the population representation criteria at that time. Thereafter, each research cycle includes $\sim 3,500$ children aged $\sim 7$ to 14 years (1993 $n=3,488,2003 n=4,095,2013 n=3,478$ ), representing $\sim 2 \%$ of Slovenia's entire population. Specific sample sizes of children and adolescents for the current study are provided in the results section under Table 1. Note that the vast majority (>99\%) of Slovenian schoolchildren attend the public-school system in the country. Private primary schools are rare and that do exist are mandated to follow the same criteria in terms of academic curricula.

\section{Data Collection}

Despite unavoidable, slight variations in data collection methods between cycles, the full dataset consists of $\geq 25$ anthropometric variables, $\geq 14$ motor and aerobic fitness variables (covering most tests from Eurofit and other test batteries from the SLOfit longitudinal database), described elsewhere (Jurak et al., 2020a). Data collection for each cycle was performed by a team of researchers well-familiarized with all test protocols. Fitness testing took place indoors (room temperature ranged between 20 and $24^{\circ} \mathrm{C}$ ), between 8:00 and 14:00 lasting 2 or 3 days for each school involved. All data collection took place in the academic fall term, from September-October. 
TABLE 1 | Descriptive characteristics stratified by age, sex, and generation.

\begin{tabular}{|c|c|c|c|c|c|c|c|}
\hline \multirow[t]{2}{*}{ Variable } & \multirow[b]{2}{*}{ Age } & \multicolumn{3}{|c|}{ Boys } & \multicolumn{3}{|c|}{ Girls } \\
\hline & & 1993 & 2003 & 2013 & 1993 & 2003 & 2013 \\
\hline \multirow{9}{*}{$\begin{array}{l}\text { Sample size } \\
\text { (N) }\end{array}$} & 7 & 167 & 235 & 203 & 197 & 229 & 236 \\
\hline & 8 & 205 & 211 & 204 & 236 & 224 & 211 \\
\hline & 9 & 233 & 232 & 195 & 212 & 224 & 180 \\
\hline & 10 & 208 & 221 & 174 & 232 & 244 & 191 \\
\hline & 11 & 199 & 242 & 168 & 201 & 226 & 186 \\
\hline & 12 & 204 & 228 & 169 & 209 & 213 & 153 \\
\hline & 13 & 214 & 236 & 141 & 224 & 204 & 142 \\
\hline & 14 & 215 & 232 & 200 & 198 & 210 & 156 \\
\hline & Total & 1645 & 1837 & 1454 & 1709 & 1774 & 1455 \\
\hline \multirow{9}{*}{$\begin{array}{l}\text { Height } \\
(\mathrm{cm})\end{array}$} & 7 & $124.3 \pm 5.5$ & $123.8 \pm 5.6$ & $125.0 \pm 5.4$ & $124.6 \pm 6.0$ & $123.6 \pm 5.9$ & $125.1 \pm 5.3$ \\
\hline & 8 & $129.9 \pm 5.6$ & $130.0 \pm 6.1$ & $131.2 \pm 5.4$ & $129.5 \pm 5.9$ & $128.9 \pm 5.7$ & $130.2 \pm 5.6$ \\
\hline & 9 & $135.8 \pm 5.6$ & $134.3 \pm 6.0$ & $136.4 \pm 5.6$ & $135.9 \pm 6.2$ & $135.5 \pm 6.0$ & $136.8 \pm 6.2$ \\
\hline & 10 & $141.3 \pm 5.8$ & $140.3 \pm 6.7$ & $142.6 \pm 7.5$ & $140.7 \pm 7.0$ & $140.1 \pm 7.0$ & $142.4 \pm 6.2$ \\
\hline & 11 & $145.8 \pm 6.5$ & $145.7 \pm 7.1$ & $148.2 \pm 7.5$ & $147.7 \pm 7.5$ & $147.2 \pm 7.1$ & $149.5 \pm 7.2$ \\
\hline & 12 & $151.9 \pm 6.5$ & $150.9 \pm 7.3$ & $153.4 \pm 7.2$ & $153.8 \pm 6.8$ & $153.8 \pm 7.0$ & $156.0 \pm 6.7$ \\
\hline & 13 & $158.2 \pm 8.4$ & $158.9 \pm 8.7$ & $160.0 \pm 8.0$ & $158.4 \pm 6.9$ & $158.3 \pm 7.5$ & $159.8 \pm 6.5$ \\
\hline & 14 & $166.7 \pm 9.2$ & $165.1 \pm 8.3$ & $167.3 \pm 7.9$ & $162.7 \pm 6.7$ & $161.1 \pm 5.9$ & $162.9 \pm 5.6$ \\
\hline & Mean & $144.4 \pm 4.0$ & $143.8 \pm 15.2^{a}$ & $145.0 \pm 15.4$ & $144.0 \pm 14.4$ & $143.0 \pm 14.3^{a}$ & $143.6 \pm 14.6$ \\
\hline \multirow{9}{*}{$\begin{array}{l}\text { Mass } \\
(\mathrm{kg})\end{array}$} & 7 & $25.2 \pm 4.7$ & $25.5 \pm 4.7$ & $25.7 \pm 4.7$ & $24.9 \pm 5.2$ & $25.6 \pm 5.5$ & $25.6 \pm 5.5$ \\
\hline & 8 & $28.0 \pm 5.4$ & $29.6 \pm 6.7$ & $29.4 \pm 5.6$ & $27.5 \pm 5.5$ & $28.6 \pm 5.9$ & $29.3 \pm 6.1$ \\
\hline & 9 & $31.9 \pm 6.5$ & $32.6 \pm 6.9$ & $33.4 \pm 6.9$ & $31.5 \pm 6.4$ & $33.0 \pm 6.7$ & $33.1 \pm 6.7$ \\
\hline & 10 & $35.1 \pm 6.8$ & $35.8 \pm 8.1$ & $38.2 \pm 8.6$ & $34.5 \pm 7.4$ & $36.0 \pm 8.1$ & $37.8 \pm 9.0$ \\
\hline & 11 & $38.6 \pm 7.9$ & $39.5 \pm 8.4$ & $42.8 \pm 10.7$ & $39.3 \pm 8.7$ & $40.8 \pm 8.4$ & $43.8 \pm 9.5$ \\
\hline & 12 & $43.4 \pm 9.4$ & $43.5 \pm 9.3$ & $46.4 \pm 10.9$ & $44.5 \pm 9.0$ & $46.8 \pm 10.2$ & $49.2 \pm 12.2$ \\
\hline & 13 & $48.5 \pm 10.6$ & $50.6 \pm 11.1$ & $52.9 \pm 13.3$ & $49.5 \pm 9.6$ & $51.7 \pm 11.1$ & $52.7 \pm 10.5$ \\
\hline & 14 & $56.6 \pm 11.9$ & $56.1 \pm 11.4$ & $59.3 \pm 12.0$ & $53.8 \pm 9.2$ & $53.9 \pm 8.5$ & $54.6 \pm 9.1$ \\
\hline & Mean & $38.5 \pm 12.9^{b, c}$ & $39.3 \pm 13.1^{a, c}$ & $40.6 \pm 14.5^{a, b}$ & $38.1 \pm 12.4^{b, c}$ & $39.1 \pm 12.7^{a, c}$ & $39.4 \pm 13.4^{a, b}$ \\
\hline \multirow{9}{*}{$\begin{array}{l}\text { BMl } \\
\left(\mathrm{kg} / \mathrm{m}^{2}\right)\end{array}$} & 7 & $16.2 \pm 2.2$ & $16.5 \pm 2.0$ & $16.4 \pm 2.3$ & $15.9 \pm 2.5$ & $16.6 \pm 2.5$ & $16.3 \pm 2.6$ \\
\hline & 8 & $16.5 \pm 2.3$ & $17.4 \pm 2.9$ & $17.0 \pm 2.5$ & $16.3 \pm 2.6$ & $17.1 \pm 2.6$ & $17.2 \pm 2.8$ \\
\hline & 9 & $17.2 \pm 2.6$ & $17.9 \pm 2.9$ & $17.8 \pm 2.8$ & $16.9 \pm 2.6$ & $17.9 \pm 2.8$ & $17.6 \pm 2.7$ \\
\hline & 10 & $17.4 \pm 2.6$ & $18.0 \pm 2.9$ & $18.7 \pm 3.2$ & $17.3 \pm 2.8$ & $18.2 \pm 3.0$ & $18.5 \pm 3.5$ \\
\hline & 11 & $18.1 \pm 3.0$ & $18.5 \pm 2.9$ & $19.3 \pm 3.7$ & $17.9 \pm 2.9$ & $18.7 \pm 2.8$ & $19.5 \pm 3.4$ \\
\hline & 12 & $18.7 \pm 3.2$ & $19.0 \pm 3.1$ & $19.6 \pm 3.6$ & $18.7 \pm 2.9$ & $19.6 \pm 3.2$ & $20.0 \pm 4.1$ \\
\hline & 13 & $19.2 \pm 2.9$ & $19.9 \pm 3.2$ & $20.5 \pm 3.9$ & $19.6 \pm 3.2$ & $20.5 \pm 3.5$ & $20.6 \pm 3.4$ \\
\hline & 14 & $20.2 \pm 3.2$ & $20.4 \pm 3.2$ & $21.1 \pm 3.4$ & $20.3 \pm 2.9$ & $20.7 \pm 2.8$ & $20.5 \pm 3.0$ \\
\hline & Mean & $18.0 \pm 3.1^{\mathrm{b}, \mathrm{c}}$ & $18.5 \pm 3.1^{a}$ & $18.7 \pm 3.6^{a}$ & $17.9 \pm 3.2^{\mathrm{b}, \mathrm{c}}$ & $18.6 \pm 3.2^{a}$ & $18.6 \pm 3.5^{a}$ \\
\hline \multirow{9}{*}{$\begin{array}{l}\text { BMI } \\
\text { (z-score) }\end{array}$} & 7 & $-0.75 \pm 0.61$ & $-0.61 \pm 0.64$ & $-0.70 \pm 0.61$ & $-0.80 \pm 0.72$ & $-0.57 \pm 0.76$ & $-0.67 \pm 0.78$ \\
\hline & 8 & $-0.60 \pm 0.67$ & $-0.34 \pm 0.82$ & $-0.42 \pm 0.79$ & $-0.61 \pm 0.76$ & $-0.49 \pm 0.71$ & $-0.40 \pm 0.75$ \\
\hline & 9 & $-0.42 \pm 0.79$ & $-0.29 \pm 0.79$ & $-0.26 \pm 0.83$ & $-0.60 \pm 0.73$ & $-0.23 \pm 0.84$ & $-0.33 \pm 0.87$ \\
\hline & 10 & $-0.33 \pm 0.74$ & $-0.20 \pm 0.86$ & $0.01 \pm 0.92$ & $-0.36 \pm 0.78$ & $-0.21 \pm 0.80$ & $0.02 \pm 0.99$ \\
\hline & 11 & $-0.16 \pm 0.97$ & $-0.09 \pm 0.80$ & $0.14 \pm 1.04$ & $-0.15 \pm 0.88$ & $0.06 \pm 0.85$ & $0.18 \pm 1.06$ \\
\hline & 12 & $-0.06 \pm 0.82$ & $0.09 \pm 0.92$ & $0.31 \pm 1.05$ & $-0.02 \pm 0.84$ & $0.19 \pm 0.90$ & $0.41 \pm 1.09$ \\
\hline & 13 & $0.19 \pm 0.88$ & $0.32 \pm 0.85$ & $0.51 \pm 1.03$ & $0.25 \pm 0.85$ & $0.54 \pm 0.90$ & $0.39 \pm 0.83$ \\
\hline & 14 & $0.40 \pm 0.92$ & $0.48 \pm 0.89$ & $0.64 \pm 0.94$ & $0.48 \pm 0.73$ & $0.52 \pm 0.82$ & $0.55 \pm 0.87$ \\
\hline & Mean & $-0.22 \pm 0.80^{\mathrm{b}, \mathrm{c}}$ & $-0.08 \pm 0.82^{a}$ & $0.03 \pm 0.91^{a}$ & $-0.23 \pm 0.79^{b, c}$ & $-0.02 \pm 0.82^{a}$ & $0.02 \pm 0.91^{a}$ \\
\hline \multirow{9}{*}{$\begin{array}{l}\text { Triceps skinfold } \\
(\mathrm{mm})\end{array}$} & 7 & $9.2 \pm 3.9^{e}$ & $10.3 \pm 3.9^{e}$ & $10.3 \pm 3.8^{\mathrm{e}}$ & $10.9 \pm 4.1^{e}$ & $12.0 \pm 4.2^{\mathrm{e}}$ & $11.8 \pm 4.4^{\mathrm{e}}$ \\
\hline & 8 & $9.5 \pm 4.0^{9}$ & $11.5 \pm 4.6^{\mathrm{g}}$ & $11.8 \pm 4.8^{g}$ & $12.0 \pm 4.7^{\mathrm{g}}$ & $12.9 \pm 4.4^{g}$ & $13.4 \pm 4.8^{g}$ \\
\hline & 9 & $11.0 \pm 5.4$ & $11.5 \pm 4.4$ & $12.2 \pm 5.0$ & $12.1 \pm 4.8$ & $14.0 \pm 4.8$ & $14.3 \pm 5.2$ \\
\hline & 10 & $11.0 \pm 5.0$ & $12.0 \pm 4.6$ & $13.1 \pm 5.4$ & $12.7 \pm 5.2$ & $13.3 \pm 4.6$ & $15.8 \pm 6.4$ \\
\hline & 11 & $11.2 \pm 5.3$ & $12.7 \pm 4.9$ & $14.2 \pm 6.6$ & $12.3 \pm 4.5$ & $13.8 \pm 4.7$ & $15.1 \pm 5.9$ \\
\hline & 12 & $11.1 \pm 5.6$ & $12.1 \pm 5.3$ & $13.6 \pm 5.4$ & $12.5 \pm 5.1$ & $13.6 \pm 4.3$ & $15.9 \pm 6.0$ \\
\hline & 13 & $10.7 \pm 5.8$ & $11.7 \pm 5.5$ & $12.8 \pm 6.1$ & $13.4 \pm 4.7$ & $14.5 \pm 4.7$ & $15.9 \pm 5.3$ \\
\hline & 14 & $10.1 \pm 5.1$ & $11.5 \pm 5.0$ & $12.0 \pm 5.4$ & $14.4 \pm 4.6$ & $14.3 \pm 4.4$ & $16.4 \pm 5.6$ \\
\hline & Mean & $10.5 \pm 5.1^{\mathrm{b}, \mathrm{c}}$ & $11.6 \pm 4.8^{\mathrm{a}, \mathrm{c}}$ & $12.4 \pm 5.4^{a, b}$ & $12.4 \pm 4.8^{\mathrm{b}, \mathrm{c}, \mathrm{d}}$ & $13.5 \pm 4.6^{a, c, d}$ & $14.6 \pm 5.6^{a, b, d}$ \\
\hline \multirow{10}{*}{$\begin{array}{l}\text { *Screen time } \\
(\min \cdot d)\end{array}$} & 7 & - & - & $107.7 \pm 64.8$ & - & - & $97.5 \pm 82.2$ \\
\hline & 8 & - & - & $121.6 \pm 85.0$ & - & - & $106.0 \pm 76.2$ \\
\hline & 9 & - & - & $130.4 \pm 83.5$ & - & - & $112.2 \pm 82.6$ \\
\hline & 10 & - & - & $148.7 \pm 102.6^{f}$ & - & - & $130.1 \pm 91.5^{f}$ \\
\hline & Mean & - & - & $126.0 \pm 85.3$ & - & - & $110.5 \pm 83.4$ \\
\hline & 11 & - & - & $289.9 \pm 187.9$ & - & - & $257.7 \pm 179.8^{d}$ \\
\hline & 12 & - & - & $321.7 \pm 190.3$ & - & - & $250.0 \pm 141.5$ \\
\hline & 13 & - & - & $334.8 \pm 162.2$ & - & - & $255.0 \pm 147.3$ \\
\hline & 14 & - & - & $347.8 \pm 191.8$ & - & - & $273.4 \pm 122.9$ \\
\hline & Mean & - & - & $330.0 \pm 184.1$ & - & - & $258.8 \pm 145.2$ \\
\hline
\end{tabular}


TABLE 1 | Continued

\begin{tabular}{|c|c|c|c|c|c|c|c|}
\hline \multirow[t]{2}{*}{ Variable } & \multirow[b]{2}{*}{ Age } & \multicolumn{3}{|c|}{ Boys } & \multicolumn{3}{|c|}{ Girls } \\
\hline & & 1993 & 2003 & 2013 & 1993 & 2003 & 2013 \\
\hline MVPA & 7 & - & - & - & - & - & - \\
\hline \multirow{8}{*}{$(\min \cdot d)$} & 8 & - & - & - & - & - & - \\
\hline & 9 & - & - & - & - & - & - \\
\hline & 10 & - & - & - & - & - & - \\
\hline & 11 & - & - & $133.1 \pm 95.2$ & - & - & $118.1 \pm 71.8$ \\
\hline & 12 & - & - & $126.1 \pm 78.9$ & - & - & $102.9 \pm 72.4^{d}$ \\
\hline & 13 & - & - & $127.7 \pm 78.6$ & - & - & $95.4 \pm 62.6^{d}$ \\
\hline & 14 & - & - & $127.7 \pm 70.1$ & - & - & $92.2 \pm 63.3^{d}$ \\
\hline & Mean & - & - & $129.8 \pm 76.4$ & - & - & $100.0 \pm 67.3$ \\
\hline Sleep & 7 & - & - & - & - & - & - \\
\hline \multirow[t]{8}{*}{ (h) } & 8 & - & - & - & - & - & - \\
\hline & 9 & - & - & - & - & - & - \\
\hline & 10 & - & - & - & - & - & - \\
\hline & 11 & - & - & $9.8 \pm 1.3^{\mathrm{e}}$ & - & - & $9.9 \pm 1.1^{\mathrm{g}}$ \\
\hline & 12 & - & - & $9.4 \pm 1.2^{\mathrm{g}}$ & - & - & $9.7 \pm 1.0^{9}$ \\
\hline & 13 & - & - & $9.0 \pm 1.2$ & - & - & $9.3 \pm 0.9$ \\
\hline & 14 & - & - & $8.9 \pm 1.1$ & - & - & $9.2 \pm 1.0$ \\
\hline & Mean & - & - & $9.3 \pm 1.2$ & - & - & $9.6 \pm 1.1$ \\
\hline
\end{tabular}

BMI, body mass index; MVPA, moderate-to-vigorous physical activity, objectively measured.

(a) Significantly different from 1993 generation, within-sex,

(b) Significantly different from 2003 generation, within-sex,

(c) Significantly different from 2013 generation, within-sex,

(d) Significantly different from boys of same generation,

(e) Significantly different from all other ages, within-sex for that generation.

(f) Significantly different than age 7-year-olds, within-sex for that generation

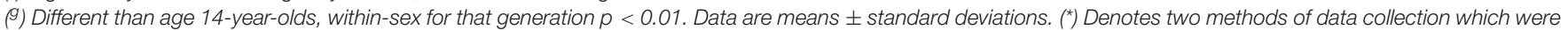

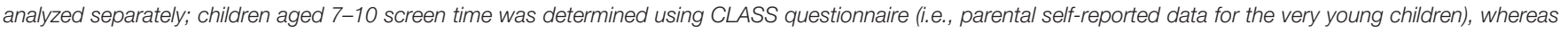
children aged 11-14 used SHAPES questionnaire, where they answered on their own behalf.

\section{Measurements}

\section{Anthropometry}

Height was measured to the nearest millimeter using a GPM 101 anthropometer (SiberHegner, Zurich, Switzerland); body mass was measured to the nearest 100 grams using a portable Tanita BWB-800P electronic scale (Arlington Heights, IL, United States). The electronic scale was calibrated each time it was moved. Skinfolds were measured to the nearest millimeter with Harpenden fat calipers (John Bull British Indicators Ltd., London, United Kingdom). Three measurements were taken at each measuring site on the right side of the body, with the mean value of the two closest measurements used for analysis.

\section{Assessment of Cardiorespiratory Fitness}

We used the Léger's original 1-min 20-m shuttle run protocol (Léger et al., 1988) which starts at a speed of $8.5 \mathrm{~km} / \mathrm{h}$ and increases by $0.5 \mathrm{~km} / \mathrm{h}$ every minute thereafter. The test was conducted indoors, in a gymnasium, with the children barefoot. Heart rate was monitored with Polar F11 heart-rate monitors (Polar Electro, Kempele, Finland) for the 2013 generation. Resting heart rate was documented at $1 \mathrm{~min}, 2.5 \mathrm{~min}$, at volitional fatigue and $5 \mathrm{~min}$ after test completion. Before commencing, an investigator explained the execution of the test to the children. Testing began with brief, light warm-up tasks (up to $10 \mathrm{~min}$ ). During the test, children were not additionally verbally encouraged. The test was terminated when the participant failed to reach the end line in time with the audio signal on two consecutive occasions, or upon volitional fatigue, whichever occurred first. CRF was estimated using the following:

$$
\dot{\mathrm{V}}{ }_{2 \text { peak }}=31.025+3.238 \cdot \mathrm{X} 1-3.248 \cdot \mathrm{X} 2+0.1536 \cdot \mathrm{X} 1 \cdot \mathrm{X} 2
$$

where $\mathrm{X} 1$ is the running speed at the last completed stage ( $\mathrm{km} /$ hour) and X2 is the age at last birthday (Léger et al., 1988).

\section{Assessment of Physical Activity, Screen Time and Sleep}

Physical activity (PA) was assessed with The School Health Action, and Evaluation System (SHAPES) (Leatherdale et al., 2009) for children aged 11-14 years, and the Children's Leisure Activities Study Survey (CLASS) printed version of parental proxy self-report questionnaire (Telford et al., 2004) for younger children aged 6-11 years. Both questionnaires were back-translated from English to Slovenian by four native Slovenian speakers, following World Health Organization recommendations for translation and adaptation of instruments (World Health Organization [WHO], 2009). Both questionnaires have acceptable reliability. Validity is more desirable in SHAPES (coeff range: 0.25-0.44) versus CLASS (coeff range: 0.02-0.82) (Adamo et al., 2009), although CLASS remains one of the best proxy instruments for younger children (Chinapaw et al., 2010). Moderate-to-vigorous PA (MVPA) was calculated based on daily self-reported MVPA minutes. Total screen time was a summation of time 
spent watching television, watching videos on computer or DVD, using cell phone, playing videogames, and browsing the internet. The Pediatric Daytime Sleepiness Scale was used to determine total sleep time in children aged 11-14 during weekdays and weekends (Drake et al., 2003). Variables (MVPA, screen time, sleep time) are calculated separately for weekdays/weekends and summed for a total minutes per week value.

\section{Statistical Analysis}

A univariate ANOVA was used to describe changes in CRF between three generations, incorporating three betweensubject factors (sex: 2 levels, male and female; generation: 3 levels, 1993, 2003, and 2013; age: 8 levels, ages 7-14, trunk age categories, e.g., 7.00-7.99 years, inclusive). Descriptive statistics on all relevant dependent measures are presented as means and standard deviations, and 95\% confidence intervals (CI) when appropriate. Post hoc analyses for multiple comparisons (Sidak, listwise) were conducted when significant main effects were found at $p<0.01$ level of significance to account for multiple comparisons. Delta change scores were calculated between the 2003 and 2013 generations for CRF and peak heart rate (within age and between sex) to determine whether changes in CRF were associated with alterations in cardiovascular strain at end stage completed. Data were initially checked for outlier analyses by examining plots visually and removing any outliers which were \pm 3.00 outside the expected range of Standard Error of Estimate in each age category, and for both sexes. Total sample size was decreased $2.4 \%$ after removing the outlier data. All numbers reported in the current manuscript reflect data after initial outlier checks. Data were analyzed using IBM SPSS v.26 (Chicago, IL, United States).

\section{RESULTS}

\section{Descriptive Characteristics}

Children differed (slightly) in height from 1993 to 2003 ( $p<0.001 ; 95 \% \mathrm{CI}: 0.4$ to $1.2 \mathrm{~cm})$, whilst mean mass was different for each generation ( $p<0.001$; 95\% CI 1993: 38.0-38.6; 2003: 39.1-39.6; 2013: 40.6-41.2 kg, Table 1). There were no differences in BMI between girls and boys $(p=0.577$; 95\% CI: $-0.15-0.083 \mathrm{~kg} \cdot \mathrm{m}^{-2}$ ) per se, although BMI did differ by generation $(p<0.001)$. Triceps thickness demonstrated a significant two-way interaction (age by sex, and generation by age, $p<0.001$, Table 1 ).

\section{0mSRT Performance Outcomes}

There were significant main effects observed for sex, generation, and age (each comparison at $p<0.001$ ), including significant two-way interactions for both sex by age and generation by age $(p<0.001)$. Post hoc comparisons indicate that each generation was unique. Boys were able to complete $\sim 1$ higher end stage than girls (boys: 4.8, 95\% CI: from 4.76 to 4.85; girls 3.9, 95\% CI from 3.85 to 3.95, $p<0.001$, Table 2). With 1993 serving as base year, end stages completed decreased by $\sim$ half a stage (95\% CI: $-0.61--0.42)$ in 2003 , before catapulting in 2013 to children completing $\sim 1$ full stage more than their 1993 counterparts (95\% CI: 0.89-1.10, $p<0.001$ ). These results translate into boys registering marginally higher $\dot{\mathrm{V}} \mathrm{O}_{2 \text { peak }}$ than girls (95\% CI: $2.1-2.4 \mathrm{~mL} \cdot \mathrm{kg}^{-1} \cdot \mathrm{min}^{-1}, p<0.001$ ) when collapsed across age and generation, and, like with end stage, each generation was unique from the other, with greatest differences occurring from 2003 to 2013 (95\% CI: $3.5-4.0 \mathrm{~mL} \cdot \mathrm{kg}^{-1} \cdot \mathrm{min}^{-1}$, $p<0.001)$. However, because there was no significant two-way interaction of sex by generation $(p=0.041)$, it can be stated that the greatest trend in this secular data is one which occurs between generations, not between the sexes. It is notable that the slope of change in $\dot{\mathrm{VO}}_{2 \text { peak }}$ is steepest in the 2003 girls' generation $\left(-0.86 \mathrm{~mL} \cdot \mathrm{kg}^{-1} \cdot \mathrm{min}^{-1}\right.$ per year $)$, which is, at minimum, three times the rate of decrease observed in boys from any generation (range: $-0.04--0.26 \mathrm{~mL} \cdot \mathrm{kg}^{-1} \cdot \mathrm{min}^{-1}$ per year).

Peak heart rate $\left(\mathrm{HR}_{\text {peak }}\right)$ was marginally lower (by $\sim 1$ beat) in boys than girls (95\% CI: 197.9-198.7 versus 199.2-200.0 b. $\left.\min ^{-1}, p<0.001\right)$; differences in $\mathrm{HR}_{\text {peak }}$ were consistently greater between-generation by $3.1-4.2$ b. $\min ^{-1}(p<0.001$, Table 2$)$. For every $\Delta \mathrm{HR}_{\text {peak }}$ of $\sim 2-6$ $\mathrm{b} \cdot \mathrm{min}^{-1}$ there was a corresponding increase $\Delta \dot{\mathrm{VO}} \mathrm{O}_{2 \text { peak }}$ of 1.5-5.0 $\mathrm{mL} \cdot \mathrm{kg}^{-1} \cdot \mathrm{min}^{-1}$ (Figure 1).

\section{Other Supporting Measures}

Children at age 7 spend $\sim 36$ fewer minutes watching screens than 10-year-olds (95\% CI: $-52.9--20.6 \mathrm{~min}, p<0.001$, Table 1). Children reported sleeping less each year, such that 11 -year-olds slept nearly 1 full hour $(\sim 0.89 \mathrm{~h})$ longer than 14 year-olds (95\% CI: $0.5-1.2 \mathrm{~h}, p<0.001)$. MVPA was lower in girls compared to boys for ages 12, 13, and 14 (each $p<0.001$; 95\% CI: 8.7-16.1 (12 year), 8.7-16.2 (13 year) and 7.7-17.2 min (14 year), respectively).

\section{Estimation of Cardiovascular Health Risk}

The 95\% CI of final stage completed for each generation was contrasted with worldwide centile normative data. Centiles varied widely between generation and sex; for example, for a given age and generation of boys, the 95\% CI fell within "very low" (0-20\%) up to "high" (60-80\%), whereas girls fared somewhat better, ranging between "low" (20-40\%) and "very high" (80-100\%, Figure 2). The proportion of children who met (or exceeded) CRF cut-off values for reduced cardiovascular health risk (boys $=41.8 \mathrm{~mL} \cdot \mathrm{kg}^{-1} \cdot \mathrm{min}^{-1}$, girls $=34.6 \mathrm{~mL} \cdot \mathrm{kg}^{-1} \cdot \mathrm{min}^{-1}$ ) were always above $50 \%$ for any given population (Figure 3 ).

\section{DISCUSSION}

This investigation found consistent declines in CRF across all ages, regardless of sex, from 1993 to 2003, before rebounding to their highest levels in 2013. Children were able to attain higher $\mathrm{HR}_{\text {peak }}$ in 2013 compared to 2003, suggesting this cohort was able to achieve, maintain and/or tolerate higher levels of cardiovascular strain at the 20mSRT endpoint. The rate of decrease in girls' CRF was worse in 2003 compared to other 
TABLE 2 | 20mSRT performance outcomes including last completed stage, estimated $\dot{V O}_{2}$ peak $\left(\mathrm{mL} \cdot \mathrm{kg}^{-1} \cdot \mathrm{min}^{-1}\right)$, and peak heart rate $(\mathrm{HR})$ at the end stage, stratified by age, sex and generation. Peak HR included for the 2003 and 2013 generations only.

\begin{tabular}{|c|c|c|c|c|c|c|c|c|c|}
\hline \multirow{2}{*}{$\begin{array}{l}\text { Age (y) } \\
\text { End Stage (\#) }\end{array}$} & \multicolumn{3}{|c|}{ All } & \multicolumn{3}{|c|}{ Boys } & \multicolumn{3}{|c|}{ Girls } \\
\hline & 1993 & 2003 & 2013 & 1993 & 2003 & 2013 & 1993 & 2003 & 2013 \\
\hline 7 & $2.0 \pm 0.8$ & $1.8 \pm 0.9$ & $3.3 \pm 1.2$ & $2.2 \pm 0.9$ & $1.9 \pm 1.0$ & $3.4 \pm 1.3$ & $1.9 \pm 0.8$ & $1.7 \pm 0.8$ & $3.2 \pm 1.1$ \\
\hline 8 & $2.8 \pm 1.3$ & $2.3 \pm 1.2$ & $3.9 \pm 1.5$ & $3.1 \pm 1.4$ & $2.4 \pm 1.3$ & $4.2 \pm 1.7$ & $2.6 \pm 1.2$ & $2.2 \pm 1.1$ & $3.7 \pm 1.4$ \\
\hline 9 & $3.2 \pm 1.6$ & $2.9 \pm 1.4$ & $4.6 \pm 1.7$ & $3.4 \pm 1.6$ & $3.1 \pm 1.6$ & $4.9 \pm 1.9$ & $3.1 \pm 1.5$ & $2.6 \pm 1.2$ & $4.3 \pm 1.4$ \\
\hline 10 & $4.0 \pm 1.7$ & $3.7 \pm 1.7$ & $4.9 \pm 1.8$ & $4.4 \pm 1.9$ & $4.2 \pm 1.8$ & $5.4 \pm 2.0$ & $3.7 \pm 1.5$ & $3.2 \pm 1.5$ & $4.5 \pm 1.6$ \\
\hline 11 & $4.7 \pm 1.9$ & $4.3 \pm 1.9$ & $5.4 \pm 2.0$ & $5.2 \pm 2.0$ & $5.0 \pm 1.9$ & $5.7 \pm 2.2$ & $4.2 \pm 1.7$ & $3.5 \pm 1.4$ & $5.1 \pm 1.7$ \\
\hline 12 & $5.3 \pm 1.8$ & $4.5 \pm 1.8$ & $6.1 \pm 2.1$ & $5.8 \pm 2.0$ & $5.1 \pm 1.9$ & $6.5 \pm 2.2$ & $4.8 \pm 1.6$ & $3.8 \pm 1.5$ & $5.6 \pm 1.8$ \\
\hline 13 & $5.6 \pm 2.0$ & $4.9 \pm 2.0$ & $6.5 \pm 2.1$ & $6.5 \pm 2.0$ & $5.5 \pm 2.1$ & $6.9 \pm 2.4$ & $4.7 \pm 1.6$ & $4.2 \pm 1.6$ & $6.0 \pm 1.8$ \\
\hline 14 & $5.9 \pm 2.2$ & $5.3 \pm 1.9$ & $6.9 \pm 2.3$ & $6.9 \pm 2.0$ & $6.1 \pm 2.0$ & $7.8 \pm 2.3$ & $4.7 \pm 1.7$ & $4.4 \pm 1.5$ & $5.9 \pm 1.8$ \\
\hline Mean total & $4.2 \pm 2.2^{b, c}$ & $3.7 \pm 2.0^{a, c}$ & $5.1 \pm 2.2^{a, b}$ & $4.7 \pm 2.4^{b, c}$ & $4.2 \pm 2.2^{a, c}$ & $5.5 \pm 2.4^{a, b}$ & $3.7 \pm 1.8^{b, c}$ & $3.2 \pm 1.6^{a, c}$ & $4.6 \pm 1.8^{a, b}$ \\
\hline \multicolumn{10}{|l|}{$\dot{\mathrm{VO}}{ }_{2 \text { peak }}\left(\mathrm{mL} \cdot \mathrm{kg}^{-1} \cdot \mathrm{min}^{-1}\right)$} \\
\hline 7 & $47.1 \pm 1.9$ & $46.6 \pm 2.0$ & $49.9 \pm 2.6$ & $47.5 \pm 2.0$ & $46.8 \pm 2.2$ & $50.2 \pm 2.9$ & $46.8 \pm 1.7$ & $46.4 \pm 1.8$ & $49.7 \pm 2.3$ \\
\hline 8 & $47.0 \pm 2.9$ & $45.9 \pm 2.6$ & $49.5 \pm 3.4$ & $47.6 \pm 3.2$ & $46.2 \pm 2.7$ & $50.1 \pm 3.6$ & $46.6 \pm 2.6$ & $45.6 \pm 2.5$ & $49.0 \pm 3.0$ \\
\hline 9 & $46.2 \pm 3.7$ & $45.4 \pm 3.3$ & $49.4 \pm 3.9$ & $46.5 \pm 3.8$ & $46.0 \pm 3.7$ & $50.0 \pm 4.4$ & $45.9 \pm 3.6$ & $44.9 \pm 2.6$ & $48.7 \pm 3.1$ \\
\hline 10 & $46.3 \pm 4.0$ & $45.5 \pm 4.0$ & $48.5 \pm 4.3$ & $47.2 \pm 4.4$ & $46.9 \pm 4.2$ & $49.5 \pm 4.7$ & $45.5 \pm 3.5$ & $44.4 \pm 3.4$ & $47.6 \pm 3.8$ \\
\hline 11 & $46.3 \pm 4.7$ & $45.2 \pm 4.5$ & $47.9 \pm 4.8$ & $47.5 \pm 4.8$ & $47.0 \pm 4.7$ & $48.6 \pm 5.4$ & $45.2 \pm 4.2$ & $43.3 \pm 3.5$ & $47.2 \pm 4.2$ \\
\hline 12 & $46.1 \pm 4.6$ & $44.1 \pm 4.6$ & $48.2 \pm 5.2$ & $47.4 \pm 5.0$ & $45.6 \pm 4.8$ & $49.3 \pm 5.6$ & $44.8 \pm 3.9$ & $42.4 \pm 3.7$ & $47.0 \pm 4.4$ \\
\hline 13 & $45.3 \pm 5.3$ & $43.5 \pm 5.2$ & $47.5 \pm 5.6$ & $47.7 \pm 5.3$ & $45.1 \pm 5.4$ & $48.7 \pm 6.1$ & $42.9 \pm 4.2$ & $41.6 \pm 4.2$ & $46.4 \pm 4.7$ \\
\hline 14 & $44.5 \pm 5.8$ & $42.8 \pm 5.2$ & $47.3 \pm 6.2$ & $47.2 \pm 5.5$ & $45.0 \pm 5.2$ & $49.5 \pm 6.2$ & $41.5 \pm 4.5$ & $40.4 \pm 4.0$ & $44.5 \pm 5.0$ \\
\hline Mean total & $46.1 \pm 4.4^{\mathrm{b}, \mathrm{c}}$ & $44.9 \pm 4.3^{a, c}$ & $48.6 \pm 4.6^{a, b}$ & $47.3 \pm 4.4^{\mathrm{b}, \mathrm{c}}$ & $46.1 \pm 4.3^{\mathrm{a}, \mathrm{c}}$ & $49.6 \pm 4.9^{a, b}$ & $44.9 \pm 4.0^{\mathrm{b}, \mathrm{c}}$ & $43.7 \pm 3.8^{a, c}$ & $47.7 \pm 4.1^{\mathrm{a}, \mathrm{b}}$ \\
\hline $\begin{array}{l}\text { Slope }\left(\mathrm{mL} \cdot \mathrm{kg}^{-1} \cdot \mathrm{min}^{-1}\right) / \\
\text { year }\end{array}$ & -0.38 & -0.54 & -0.37 & -0.04 & -0.26 & -0.09 & -0.76 & -0.86 & -0.75 \\
\hline \multicolumn{10}{|l|}{ Peak Heart Rate (b.min $\left.{ }^{-1}\right)$} \\
\hline 7 & - & $193 \pm 14$ & $199 \pm 12$ & - & $193 \pm 11$ & $199 \pm 14$ & - & $193 \pm 17$ & $199 \pm 11$ \\
\hline 8 & - & $193 \pm 15$ & $199 \pm 11$ & - & $191 \pm 14$ & $199 \pm 11$ & - & $195 \pm 15$ & $199 \pm 11$ \\
\hline 9 & - & $197 \pm 11$ & $200 \pm 14$ & - & $196 \pm 11$ & $200 \pm 13$ & - & $198 \pm 10$ & $201 \pm 14$ \\
\hline 10 & - & $198 \pm 11$ & $201 \pm 10$ & - & $198 \pm 12$ & $201 \pm 10$ & - & $199 \pm 9$ & $202 \pm 10$ \\
\hline 11 & - & $199 \pm 11$ & $201 \pm 11$ & - & $199 \pm 10$ & $200 \pm 12$ & - & $199 \pm 12$ & $202 \pm 10$ \\
\hline 12 & - & $199 \pm 10$ & $202 \pm 10$ & - & $199 \pm 9$ & $201 \pm 11$ & - & $199 \pm 10$ & $204 \pm 9$ \\
\hline 13 & - & $200 \pm 10$ & $202 \pm 10$ & - & $199 \pm 9$ & $200 \pm 12$ & - & $201 \pm 11$ & $203 \pm 9$ \\
\hline 14 & - & $198 \pm 10$ & $201 \pm 11$ & - & $198 \pm 10$ & $201 \pm 10$ & - & $199 \pm 10$ & $201 \pm 11$ \\
\hline Mean & - & $197 \pm 12$ & $201 \pm 11^{b}$ & - & $197 \pm 11$ & $200 \pm 12^{b}$ & - & $198 \pm 12^{d}$ & $201 \pm 11^{b, d}$ \\
\hline
\end{tabular}

"Slope" refers to the rate of reduction in $\mathrm{V}_{2}$ peak per year from age 7 to 14 for that sex, within that generation.

(a) Significantly different from 1993 generation, within-sex,

(b) Significantly different from 2003 generation, within-sex,

(c) Significantly different from 2013 generation, within-sex,

(d) Significantly different from boys of same generation.

generations. Slovenian schoolchildren achieve minimum CRF cut-off point to avoid cardiovascular health risks, ranging from 75 to $100 \%$ for any given generation.

\section{Secular Trends in Slovenia}

Cardiorespiratory fitness decreased by 2.8\% from 1993 to 2003 for both boys and girls, as estimated by the original Léger equation. There is continued debate on the best, or rather, most accurate model to assess 20mSRT as a predictor of CRF (Welsman and Armstrong, 2019). Knowing these limitations, it remains that initial decreases in CRF were reversed from 2003 to $2013(\sim 8.2 \%)$, across all ages, in both sexes and inline with overall secular trends of physical fitness in Slovenia during that time (Potočnik et al., 2020). Indeed, CRF increased $\sim 5.4 \%$ across the 20-year (1993-2013) period of study. The rate of reduction in CRF from age 7 to 14 (i.e., the slope of the response) was lowest in 2013 (i.e., more favorable). Since participants must move their entire body mass against the forces of gravity, we report $20 \mathrm{mSRT}$ relative to total body mass of the individual. Emerging research is considering updating the original Léger equation in favor of a curvilinear allometric model (Nevill et al., 2020). To what extent new models may impact calculated risk assessments, centile normative values, or test interpretation, remains unclear. What is clear from this reporting is that, at a population level, the CRF of Slovenian schoolchildren remain relatively high, possibly since Slovenia maintains some of the highest levels of child PA in the world (Sember et al., 2018). 

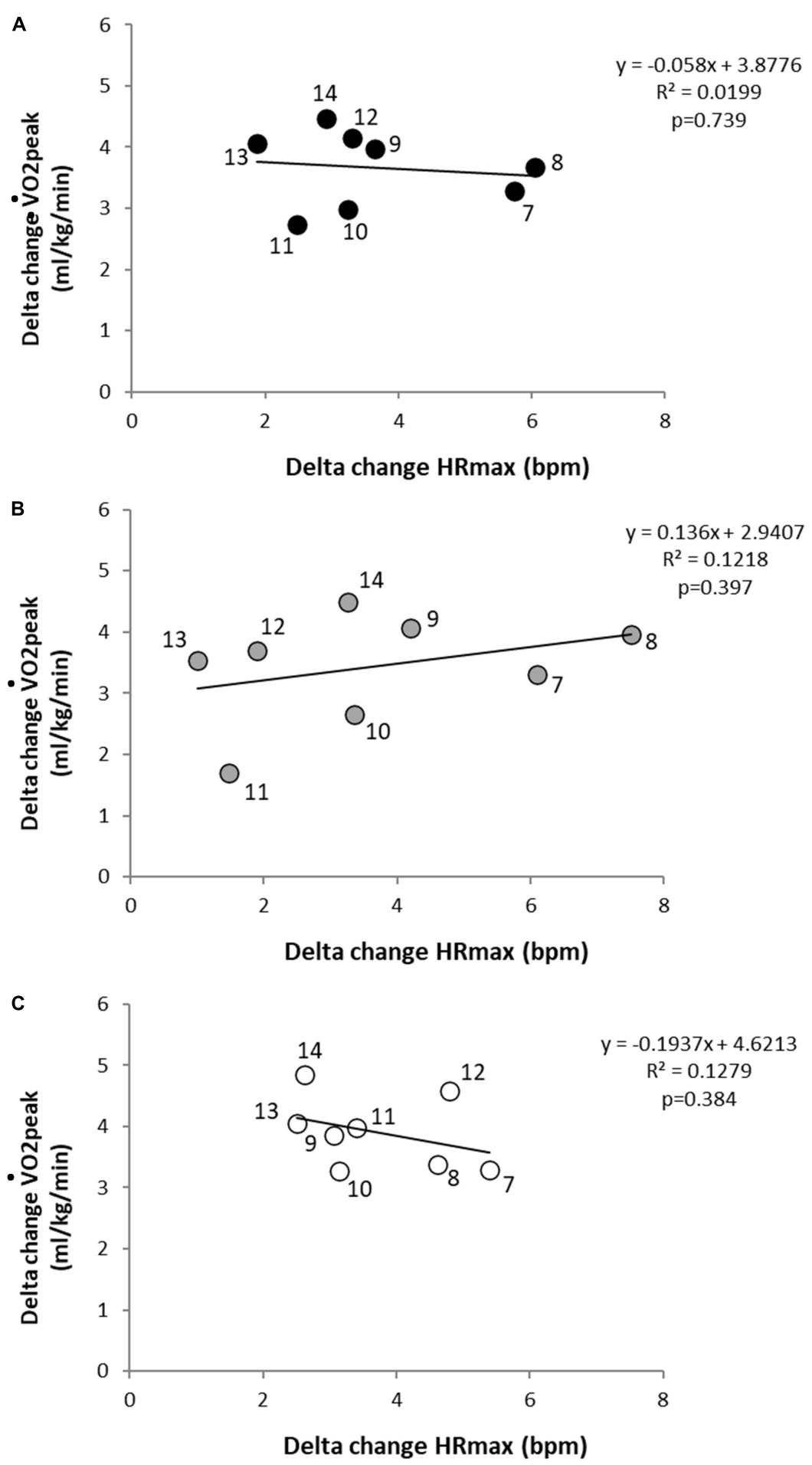

FIGURE 1 | Delta change scores between the 2003 and 2013 generations in aerobic fitness and maximum heart rate for (A) all (B) boys, and (C) girls. Scatterplots depict mean change for a given age group (age labeled beside each dot).

\section{Possible Drivers of Generational Change in CRF}

Between 1993 and 2003, CRF in Slovenian schoolchildren was systematically worsening, reflecting global trends for this metric
(Tomkinson et al., 2003), likely due to several factors. For one, changes in Slovenia's political system (especially since 1991) affected family economics such that economically weaker ones could not enroll children in expensive sporting programmes 

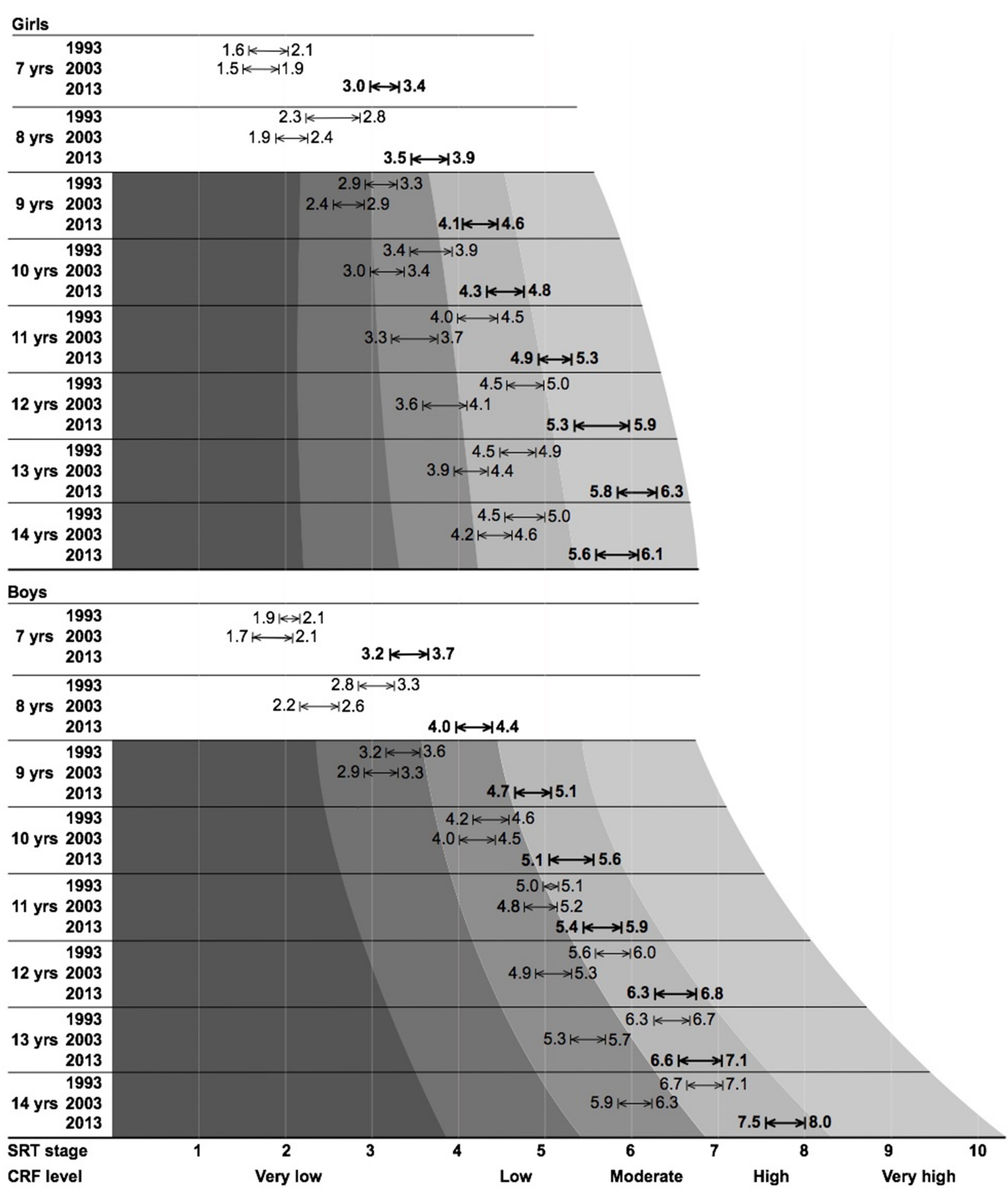

FIGURE 2 | Final stage completed for each generation of Slovenian schoolchildren, contrasted with worldwide centile normative data (where available). Values listed correspond to the lower- and upper-bound 95\% confidence intervals.

(Jurak et al., 2002a). Additionally, the sheltering praxis of parents promoted restricting child access to public spaces (e.g., playing on playgrounds, walking alone), individualization, and sedentary lifestyle each became commonplace amongst Slovenian children (Starc and Kovač, 2007). Moreover, it was found that physical education (PE) teachers were omitting some physically intensive content in their classes (Bučar Pajek et al., 2010), and these PE lessons (especially at lower ages) were often taught by general practitioners less effective in class organization (Jurak et al., 2006). With a mean sleep time of $9.4 \pm 1.2 \mathrm{~h}$, children are generally achieving enough cumulative sleep to meet international standards for that metric (Paruthi et al., 2016) 

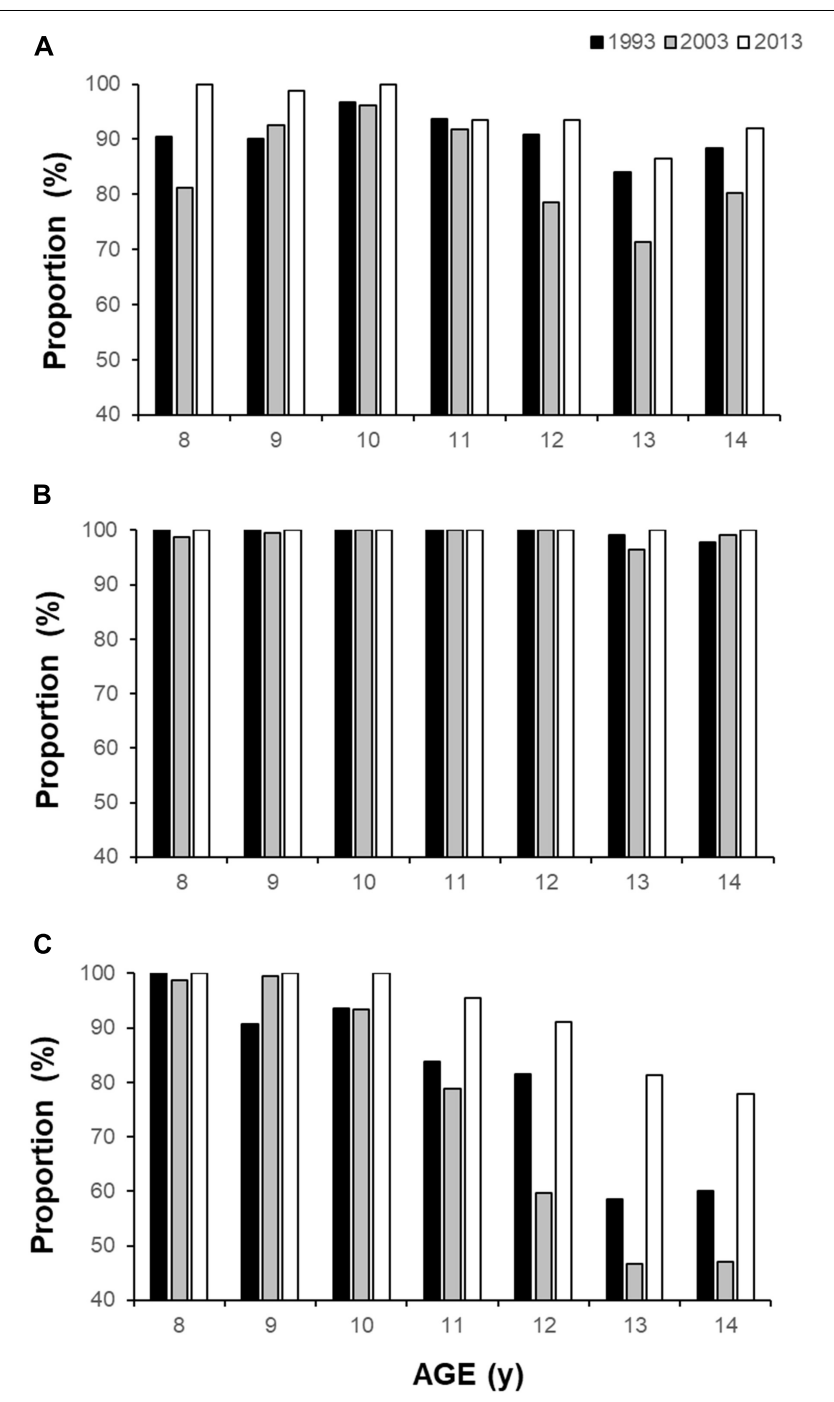

FIGURE 3 | Proportion of children who meet (or exceed) the CRF cut-off value for reduced health risk for (A) boys $\left(41.8 \mathrm{ml} \cdot \mathrm{kg}^{-1} \cdot \mathrm{min}^{-1}\right)$, (B) girls $\left(34.6 \mathrm{ml} \cdot \mathrm{kg}^{-1} \cdot \mathrm{min}^{-1}\right)$, and $\mathbf{( C )}$ proportion of girls who meet or exceed the boys' cut-off standard, stratified by age and generation.

and was not considered a factor in the generational changes observed in CRF.

There was a combination of societal changes in the 2000's, including several systematic interventions at the national level, that did contribute to more favorable trends in PA, which is reflected in the improved CRF scores from 2003 to 2013. Namely, this period was marked by the implementation of new PE curricula which provided almost all schoolchildren (aged 6-14 year) with 135 min of PE per week, delivered by specialist PE teachers from age 11 onwards, and taught separately by gender (Jurak et al., 2020b). Furthermore, because increasing trends in childhood obesity and declining fitness across several metrics were observed within the national child physical fitness surveillance system (SLOfit) (Jurak et al., 2020a), a national PA intervention program entitled "Healthy Lifestyle" (HLS) was implemented from 2010/11, which provided two additional PE hours per week, delivered by specialist physical educators for students 6-14 years old (Jurak et al., 2020b). HLS was considered part of a school's extracurricular health-oriented PA program and included more than $30 \%$ of the entire elementary school population during its tenure. HLS was a coordinated effort by the national government to inject greater PA volume into the school system, ultimately exposing children to up to $51 \mathrm{~min}$ of daily PA (Sember et al., 2016) they would otherwise not have received.

\section{Normative Reference Standards for Estimating Health Risk as a Pedagogical Tool for Physical Literacy}

Health practitioners, clinicians and teachers propose applying international standards of CRF more readily in clinical and school settings (Lang et al., 2019). Normative CRF data, similar to international BMI growth curves developed for children and youth (Ruiz et al., 2016), can be a helpful tool to identify children who need to increase PA, improve overall health, and reduce their risk of future disease-state outcomes. For practical use, CRF standards-based feedback should provide experts with a comprehensive way to increase the physical literacy of students and their parents (Whitehead, 2001). Based on the results of this study, the question arises as to whether existing CRF normativereferenced standards for estimating health risk (Ruiz et al., 2016) fulfill this role. In particular, almost all Slovenian schoolchildren herein meet international guidelines for healthy CRF. However, when examining the results in detail, Slovenia can have 9-year-old boys in the "very low" to "low" centile range, but $80-90 \%$ of them are achieving the cut-off point for low cardiovascular risk. This discrepancy is exacerbated in girls such that, for any generation, nearly $100 \%$ of all girls met the published minimum health risk standards. Such discrepancies are also common in populations that are less fit (Tomkinson et al., 2018). Indeed, having a minimum standard of $9.0 \mathrm{~km} / \mathrm{h}$ (especially in the younger age groups) is a somewhat controversial, since the 20mSRT test starts at a speed of $8.5 \mathrm{~km} / \mathrm{h}$, children need only complete one stage to achieve a "low-risk" health status. The authors posit that perhaps we are entering a new phase of 20mSRT fitness testing; in general, it has proven that when measured correctly the test is reliable and valid, and research groups are continuing to hone the predictive model validity of the measure (Nevill et al., 2020), but now we must harmonize these research findings to a more meaningful pedagogical and translational public health tool.

\section{Strengths, Weaknesses and Study Considerations}

The 20mSRT was measured at the same locations for each iteration of data collection. Despite this, there are many factors that can affect test performance, including: environmental conditions, clothing, field surfaces, footwear, motivation, pretest instructions, and diurnal variation. The current study makes every effort to standardize these factors. Testing took place in the fall semester, indoors, in a gym, with children wearing light, athletic clothing, barefoot, having standard pretest instructions, and always in the morning. Standardized 
fitness testing is a common and consistent tool measured annually in Slovenia [SLOfit (Jurak et al., 2020a)], so although there are likely motivational factors which vary across individual schoolchildren, they are nonetheless accustomed to systematic fitness testing in schools. Peak heart rate was available only during the 2003 and 2013 generation. A 20mSRT data were recorded as last stage completed, not consistently in total laps. We therefore calculated speed at last stage and estimated $\dot{\mathrm{V}} \mathrm{O}_{2 \text { peak }}$ but were not confident in reporting total laps for all generations herein. Involvement in this study was voluntary and based on informed parental consent. Since the physical fitness test could represent a risk to children with certain chronic diseases, only healthy children without injuries were included in the study. It is not possible to assess the reasons why parents might not have given positive consent for their children which would have resulted in rejection of their child's participation. Among those (few) parents who did opt out, it is possible the reason could have been due to certain chronic or acute health conditions, but since there is no personal data to consider, we cannot speculate on any possible difference(s) between children included in this study, and those who did not participate.

\section{CONCLUSION}

Negative trends in CRF from Slovenian schoolchildren were reversed by 2013, indicating that Slovenia should continue implementing the progressive national physical fitness strategies introduced between sampling years (e.g., 2003-2013). Additionally, due to the universal nature of Slovenian schoolchildren achieving the cut-off points for reduced health risk, it is proposed that more specific benchmark criteria are developed so the results can be more accurately utilized in clinical and pedagogical settings. Cut-off values for healthy zones in younger ages should be revised inline with more modern methods of calculating CRF. Further investigations of CRF secular trends may include accounting for generation changes in mass, maturation, body composition and other environmental factors known to significantly affect performance outcomes.

\section{DATA AVAILABILITY STATEMENT}

The raw data supporting the conclusions of this article will be made available by the authors, without undue reservation.

\section{REFERENCES}

Adamo, K. B., Prince, S. A., Tricco, A. C., Connor-Gorber, S., and Tremblay, M. S. (2009). A comparison of indirect versus direct measures for assessing physical activity in the pediatric population: a systematic review. Int. J. Paediatr. Obes. 4, 2-27. doi: 10.1080/17477160802315010

Armstrong, N., and Welsman, J. (2019). Twenty-metre shuttle run: (mis)representation, (mis)interpretation and (mis)use. Br. J. Sports Med. 53:1199. doi: 10.1136/bjsports-2018-100082

\section{ETHICS STATEMENT}

The studies involving human participants were reviewed and approved by the Slovenia National Medical Ethics Committee (ID: 138/05/13). Written informed consent to participate in this study was provided by the participants' legal guardian/next of kin.

\section{AUTHOR CONTRIBUTIONS}

SM: conceptualization, formal analysis, visualization, writing original draft, writing, review, and editing. VS: investigation, data curation, formal analysis, and writing - original draft. BL: data curation, formal analysis, writing, review, and editing. MK: investigation, resources, writing, review, editing, and project administration. GJ and GS: conceptualization, investigation, resources, writing, review, editing, and project administration. All authors contributed to the article and approved the submitted version.

\section{FUNDING}

Limited non-specific funding was provided by the Slovenian National Research Agency (P5-0142 Bio-psycho-social context of kinesiology).

\section{ACKNOWLEDGMENTS}

This study received valuable assistance from the Slovenian Olympic Committee and Elan Inventa, a sporting equipment manufacturer and supplier. We thank the patrons who supported this study: the Slovenian Federation of Sport Teacher Associations, the Section for School and High-school Medicine at the Slovenian Medical Association, the Ministry of Health of the Republic of Slovenia, the Ministry of Education, Science and Sport of Slovenia, the Olympic Committee of Slovenia. A special thanks to Prof. Emeritus Janko Strel, the previous principal investigator of the ACDSi study, for his valuable legacy and knowledge transfer of the study's infrastructure. Appreciation is expressed to Dr. Justin Lang, Public Health Agency of Canada, for his valuable feedback on manuscript preparation. We thank the voluntary investigators, students, researchers, school coordinators, principals, children, and parents involved in this ongoing, multidisciplinary study.

Bučar Pajek, M., Čuk, I., Kovač, M., and Jakše, B. (2010). Implementation of the gymnastics curriculum in the third cycle of basic school in Slovenia. Sci. Gymnast. J. 2, 15-27.

Chinapaw, M. J. M., Mokkink, L. B., van Mechelen, W., and Terwee, C. B. (2010). Physical activity questionnaires for youth: a systematic review of measurement properties. Sports Med. 40, 539-563. doi: 10.2165/11530770-00000000000000

Drake, C., Nickel, C., Burduvali, E., Roth, T., Jefferson, C., and Badia, P. (2003). The pediatric daytime sleepiness scale (PDSS): sleep habits and school 
outcomes in middle-school children. Sleep 26, 455-458. doi: 10.1037/t027 61-000

García-Hermoso, A., Ramírez-Vélez, R., García-Alonso, Y., Alonso-Martínez, A., and Izquierdo, M. (2020). Association of cardiorespiratory fitness levels during youth with health risk later in life: a systematic review and metaanalysis. JAMA Pediatr. 174, 952-960. doi: 10.1001/jamapediatrics.2020. 2400

Jurak, G., Kovač, M., and Starc, G. (2013). The ACDSi 2013 - the analysis of children's development in slovenia 2013: study protocol. Anthropol. Notebooks 19, 123-143.

Jurak, G., Kovac, M., and Strel, J. (2002a). How Slovene primary school pupils spend their summer holidays. Kinesiol. Slov. 8, 35-43.

Jurak, G., Kovač, M., and Strel, J. (2006). Impact of the additional physical education lessons programme on the physical and motor development of 7 to 10-year-old children. Kinesiology 38, 105-115.

Jurak, G., Leskošek, B., Kovac, M., Soric, M., Kramaršič, J., Sember, V., et al. (2020b). SLOfit surveillance system of somatic and motor development of children and adolescents: upgrading the Slovenian Sports Educational Chart. Kinanthropologica 56, 28-40. doi: 10.14712/23366052.2020.4

Jurak, G., Starc, G., and Kovač, M. (2020a). "Physical education and school sport in Slovenia," in Research on Physical Education and School Sport in Europe, eds R. Naul and C. Scheuer (Osnabrück: Meyer \& Meyer).

Kovač, M., Starc, G., and Pajek, M. B. (2004). Analiza Nekaterih Povezav Gibalnih Sposobnosti in Telesnih Znaèilnosti Z Drugimi Razsežnostmi Psihosomatiènega Statusa Slovenskih Otrok in Mladine [Analysis of Some Correlations Between Motor Abilities, Physical Characterisitcs and Other Dimensions of Psychosomatic Status of Slovenian Children and Youth]. Ljubljana: Institute of Kinesiology Faculty of Sport.

Lang, J., Tomkinson, G., Janssen, I., Ruiz, J., Ortega, F., Léger, L., et al. (2018a). Making a case for cardiorespiratory fitness surveillance among children and youth. Exerc. Sport Sci. Rev. 46, 66-75. doi: 10.1249/JES.000000000000 0138

Lang, J., Tremblay, M., Léger, L., Olds, T., and Tomkinson, G. (2018b). International variability in $20 \mathrm{~m}$ shuttle run performance in children and youth: who are the fittest from a 50-country comparison? A systematic literature review with pooling of aggregate results. Br. J. Sports Med. 52, 276. doi: 10. 1136/bjsports-2016-096224

Lang, J., Tremblay, M., Ortega, F., Ruiz, J., and Tomkinson, G. (2019). Review of criterion-referenced standards for cardiorespiratory fitness: what percentage of 1142026 international children and youth are apparently healthy? Br. J. Sports Med. 53, 953-958. doi: 10.1136/bjsports-2016-09 6955

Leatherdale, S. T., Manske, S., Wong, S. L., and Cameron, R. (2009). Integrating research, policy, and practice in school-based physical activity prevention programming: the School Health Action, Planning, and Evaluation System (SHAPES) Physical Activity Module. Health Promot. Pract. 10, 254-261. doi: 10.1177/1524839906298499

Léger, L., Lambert, J., Goulet, A., Rowan, C., and Dinelle, Y. (1984). Aerobic capacity of 6 to 17-year-old Quebecois-20 meter shuttle run test with 1 minute stages [Article in French]. Can. J. Appl. Sport Sci. 9, 64-69.

Léger, L., Mercier, D., Gadoury, C., and Lambert, J. (1988). The multistage 20meter shuttle run test for aerobic fitness. J. Sports Sci. 6, 93-101. doi: 10.1080/ 02640418808729800

Machado-Rodrigues, A., Leite, N., Coelho-e-Silva, M., Martins, R., Valentedos-Santos, J., Mascarenhas, L., et al. (2014). Independent association of clustered metabolic risk factors with cardiorespiratory fitness in youth aged 11-17 years. Ann. Hum. Biol. 41, 271-276. doi: 10.3109/03014460.2013.85 6471

NCD Risk Factor Collaboration [NCD-RisC] (2017). Worldwide trends in bodymass index, underweight, overweight, and obesity from 1975 to 2016: a pooled analysis of 2416 population-based measurement studies in 128.9 million children, adolescents, and adults. Lancet 390, 2627-2642.

Nevill, A. M., Ramsbottom, R., Sandercock, G., Bocachica-González, C. E., Ramírez-Vélez, R., and Tomkinson, G. (2020). Developing a new curvilinear allometric model to improve the fit and validity of the $20-\mathrm{m}$ shuttle run test as a predictor of cardiorespiratory fitness in adults and youth. Sports Med. [Epub ahead of print]. doi: 10.1007/s40279-020-01346-0
Olds, T., Tomkinson, G., Léger, L., and Cazorla, G. (2006). Worldwide variation in the performance of children and adolescents: an analysis of 109 studies of the $20-\mathrm{m}$ shuttle run test in 37 countries. J. Sports Sci. 24, 1025-1038. doi: $10.1080 / 02640410500432193$

Paruthi, S., Brooks, L., D’Ambrosio, C., Hall, W., Kotagal, S., Lloyd, R., et al. (2016). Consensus statement of the American Academy of Sleep Medicine on the recommended amount of sleep for healthy children: methodology and discussion. J. Clin. Sleep Med. 12, 1549-1561. doi: 10.5664/jcsm. 6288

Potočnik ŽL, Jurak, G., and Starc, G. (2020). Secular trends of physical fitness in twenty-five birth cohorts of slovenian children: a population-based study. Front. Public Health 8:561273. doi: 10.3389/fpubh.2020.561273

Ramírez-Vélez, R., Palacios-López, A., Humberto Prieto-Benavides, D., Enrique Correa-Bautista, J., Izquierdo, M., Alonso-Martínez, A., et al. (2017). Normative reference values for the $20 \mathrm{~m}$ shuttle-run test in a population-based sample of school-aged youth in Bogota, Colombia: the FUPRECOL study. Am. J. Hum. Biol. 29:e22902. doi: 10.1002/ajhb.22902

Ruiz, J., Cavero-Redondo, I., Ortega, F., Welk, G., Andersen, L., and MartinezVizcaino, V. (2016). Cardiorespiratory fitness cut points to avoid cardiovascular disease risk in children and adolescents; what level of fitness should raise a red flag? A systematic review and meta-analysis. Br. J. Sports Med. 50, 1451-1458. doi: 10.1136/bjsports-2015-095903

Sember, V., Morrison, S. A., Jurak, G., Kovač, M., Golobič, M., Pavletić Samardžija, P., et al. (2018). Results from Slovenia's 2018 report card on physical activity for children and youth. J. Phys. Act. Health 15, S404-S405. doi: 10.1123/jpah.20180542

Sember, V., Starc, G., Jurak, G., Golobič, M., Kovač, M., Samardžija, P. P., et al. (2016). Results from the Republic of Slovenia's 2016 report card on physical activity for children and youth. J. Phys. Act. Health 13(11 Suppl. 2), S256-S264. doi: 10.1123/jpah.2016-0294

Sorić, M., Jurak, G., Đurić, S., Kovač, M., Strel, J., and Starc, G. (2020). Increasing trends in childhood overweight have mostly reversed: 30 years of continuous surveillance of Slovenian youth. Sci. Rep. 10:11022. doi: 10.1038/s41598-02068102-2

Starc, G., and Kovač, M. (2007). “Življenjski slogi otrok in mladine med izbiro in določenostjo," in Šport in Življenjski Slogi Slovenskih Otrok in Mladine, eds G. Starc and M. Kovač (Ljubljana: Fakulteta za šport, Inštitut za kineziologijo), 29-34.

Strel, J., Šturm, J., Štihec, J., Kovaè, M., Tušak, M., Ambrožič, F., et al. (1996). Analiza Razvojnih Trendov Motoriènih Sposobnosti in Morfoloških Znaèilnosti in Relacij Obeh s Psihološkimi in Sociološkimi Razsežnostmi Slovenskih Otrok in Mladine Med 7.-18. Letom Starosti v Obdobju 1970-1983-1993. Zakljuèno Poroèilo [Analysis of Developmental Trends in Motor Abilities and Morphological Characteristics and Relations of Both Dimensions to Psychological and Sociological Dimensions of Slovenian Children and Youth Aged 7-18 Years in Period 1970-1983-1993. Final Report]. Ljubljana: Faculty of Sport.

Šturm, J., and Strel, J. (1984). Primerjava Nekaterih Motoriènih in Morfoloških Parametrov v Osnovnih Šolah SR Slovenije v Obdobju 1970/71-83. Zakljuèno Poroèilo [Comparison of Certain Motor and Morphological Parameters in Elementary Schools in Slovenia in Period 1970/71-83. Final Report]. Ljubljana: Faculty of Physical Culture.

Telford, A., Salmon, J., Jolley, D., and Crawford, D. (2004). Reliability and validity of physical activity questionnaires for children: the Children's Leisure Activities Study Survey (CLASS). Pediatr. Exer. Sci. 16, 64-78. doi: 10.1123/pes. 16.1 .64

Tomkinson, G., Carver, K., Atkinson, F., Daniell, N., Lewis, L., Fitzgerald, J., et al. (2018). European normative values for physical fitness in children and adolescents aged 9-17 years: results from 2779165 Eurofit performances representing 30 countries. Br. J. Sports Med. 52, 1445-14563. doi: 10.1136/ bjsports-2017-098253

Tomkinson, G., Lang, J., Blanchard, J., Léger, L., and Tremblay, M. (2019a). The 20$\mathrm{m}$ shuttle run: assessment and interpretation of data in relation to youth aerobic fitness and health. Pediatr. Exer. Sci. 31, 152-163. doi: 10.1123/pes.2018-0179

Tomkinson, G., Lang, J., and Tremblay, M. (2019b). Temporal trends in the cardiorespiratory fitness of children and adolescents representing 19 highincome and upper middle-income countries between 1981 and 2014. Br. J. Sports Med. 53, 478-486. doi: 10.1136/bjsports-2017-097982 
Tomkinson, G., Léger, L., Olds, T., and Cazorla, G. (2003). Secular trends in the performance of children and adolescents (1980-2000): an analysis of 55 studies of the $20 \mathrm{~m}$ shuttle run test in 11 countries. Sports Med. 33, 285-300. doi: 10.2165/00007256-200333040-00003

Welsman, J., and Armstrong, N. (2019). The $20 \mathrm{~m}$ shuttle run is not a valid test of cardiorespiratory fitness in boys aged 11-14 years. BMJ Open Sport Exerc. Med. 5:e000627. doi: 10.1136/bmjsem-2019-000627

Whitehead, M. (2001). The concept of physical literacy. Eur. J. Phys. Educ. 6, 127-138. doi: 10.1080/1740898010060205

World Health Organization [WHO] (2009). Process of Translation and Adaptation of Instruments. Geneva: World Health Organization.
Conflict of Interest: The authors declare that the research was conducted in the absence of any commercial or financial relationships that could be construed as a potential conflict of interest.

Copyright (c) 2021 Morrison, Sember, Leskošek, Kovač, Jurak and Starc. This is an open-access article distributed under the terms of the Creative Commons Attribution License (CC BY). The use, distribution or reproduction in other forums is permitted, provided the original author(s) and the copyright owner(s) are credited and that the original publication in this journal is cited, in accordance with accepted academic practice. No use, distribution or reproduction is permitted which does not comply with these terms. 